\title{
Novel Core Designs to Miniaturise Passive Magnetic Components
}

\author{
Adam Walker, Gaurang Vakil, Christopher Gerada \\ Power Electronics Machines and Control Group \\ University of Nottingham \\ Nottingham, UK \\ Adam.Walker@nottingham.ac.uk
}

\begin{abstract}
Converter sizes are often dominated by passive magnetic components. Passive magnetic component sizes are in turn limited by electromagnetic saturation and thermal limits. This paper covers two modifications to the air gaps of tape wound amorphous magnetic cores, made available by the advancement of additive manufacturing with magnetic materials. These are modifying the width of the core at the air gap and moving the air gap to a different location in the core. The objective was to reduce the effect of fringing flux, and to overcome the issue of a high loss zone and thermal hot spot being generated at the air gap, situated under the windings. These modifications will reduce temperatures and thus allow for smaller components before thermal limits are reached. The changes this causes to inductance, flux density and losses are highlighted and discussed.
\end{abstract}

Keywords-Magnetic core design, Magnetic losses, Passive magnetic components, Inductors

\section{INTRODUCTION}

Magnetic components are required in a wide range of applications, from automotive to aerospace to power generation. These components often contribute a significant portion of a converters mass and volume, potentially larger than $50 \%[1,2]$.

A key issue restricting the size of magnetic components is the restriction of selection in off the shelf cores available. The cores typically come in fixed sizes and generic shapes - toroid, C cores and E cores. The differences between one size of core and the next can be significant, amorphous cores are typically available in the steps of $10 \%$ [3], while speciality iron powder cores can have a $30 \%$ size increment between cores [4]. If the performance requirement of an inductor necessitates a core size which is not readily available the closest purchasable option must be used. However this closest option must be larger than the calculated requirement, to ensure the core does not saturate or overheat, leading to the development of an oversized magnetic component.

Innovation in core design is restricted by the off the shelf available cores. Bespoke prototypes outside these fixed sizes and shapes are expensive to produce, typically several orders of magnitude higher than off the shelf cores. This price difference limits investigation into novel core shapes. A moderate increase in performance is often not justifiable for a drastic increase in price. Which in turn, restricts any new core shapes being mass produced, as there is no evidence they would be beneficial. While laminated cores can be cut to shape, at high frequencies amorphous or iron powder cores must be used to minimise the

Work funded by EPSRC Challenge Network in Automotive Powe Electronics Grant on Miniaturising Magnetics Through 3D Design and Manufacture (Mini Mags). losses. Machining amorphous and iron powder cores postmanufacture leads to several difficulties, the brittleness of the material causing issues during the process and even successful cuts resulting a high loss zone along the machined edge. This has recently been counteracted by the potential use of additive manufacturing of magnetic cores [5-7]. This will allow for bespoke cores to be produced with reasonable costs and times. A typical problem when trying to miniaturise inductors is that a large portion of the losses are produced at the air gap. These losses then cause a hot spot, and because this air gap is under the windings it is difficult to build an effective thermal pathway to it [8]. This leads to a thermal limitation in the core, restricting the miniaturisation of magnetic components. Using additive manufacturing the core shapes can be modified to overcome this issue. This paper will focus on these modifications of the core shape for a specific application, presented in section II, investigating how the losses can be reduced and the thermal management improved. Analytical models validated with finite element analysis are used for the electromagnetic design and simulation. Lumped parameter thermal networks are used for the thermal analysis.

\section{BACKGROUND}

Considered here is a case study of amorphous $\mathrm{C}$ cores inductors, originally designed for an automotive DC-DC converter for the project 'Frequent Integrated Robust Soft Stop Start Technology' (FIRS3T). The project was aimed at designing a motor along with the power electronics and energy storage for a hybrid electric bus. This specific inductor was to provide current smoothing for the energy storage system, as shown in Fig. 1. The performance requirements are given in TABLE I and the details conventional inductor deigned are in TABLE II.

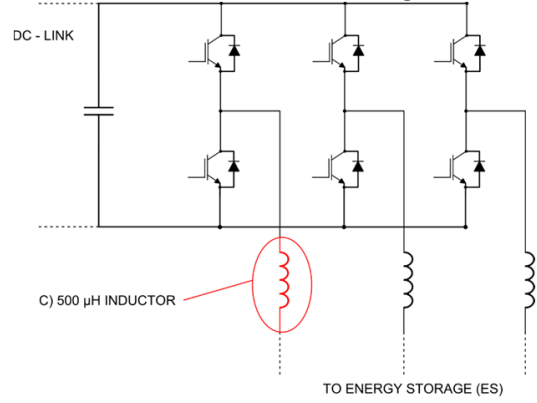

Fig. 1. Vehicle energy storage inverter 
TABLE I INDUCTOR PERFORMANCE REQUIRMENTS

\begin{tabular}{|l|c|}
\hline Idc rated $(\mathrm{A})$ & 20 \\
\hline Idc peak $(\mathrm{A}) 1 \mathrm{~second}$ & 42 \\
\hline Ipk2pk $(\mathrm{A})$ & 8.5 \\
\hline Frequency $(\mathrm{kHz})$ & 50 \\
\hline Vdc $(\mathrm{V})$ & 800 \\
\hline Inductance $(\mu \mathrm{H})$ & 500 \\
\hline Ambient temperature $\left({ }^{\circ} \mathrm{C}\right)$ & 105 \\
\hline
\end{tabular}

TABLE II ORIGINAL INDUCTOR DETAILS

\begin{tabular}{|l|l|}
\hline Core & AMCC40 \\
\hline Material & Metglas Alloy 2605SA1 \\
\hline Turns & 38 \\
\hline Conductor Width $(\mathrm{mm})$ & 2 \\
\hline Conductor height $(\mathrm{mm})$ & 3.55 \\
\hline Mean Length Turn $(\mathrm{mm})$ & 108 \\
\hline DC Resistance $(\Omega)$ & 0.0097 \\
\hline
\end{tabular}

\section{CONCEPT}

Using the benefits provided by additive manufacturing cores two avenues of investigation will be considered. The first is modifying the area of the air gap to minimise the fringing flux. The other is moving the placement of the air gap within the core, with the goal of negating the fringing flux and moving the high loss zone to a more thermally accessible area. To help with understanding, in Fig. 2, areas and measures of the new cores are displayed.

1. Tooth

2. Tooth Tip - the area of the tooth adjacent to the air gap.

3. Slot Length - the longer side of the core

4. Slot Width - the shorter side of the core

5. Air gap placement offset - the distance from the base of the slot length to the air gap.

6. Positive air gap width offset - width of material added

7. Negative air gap width offset - width of material removed
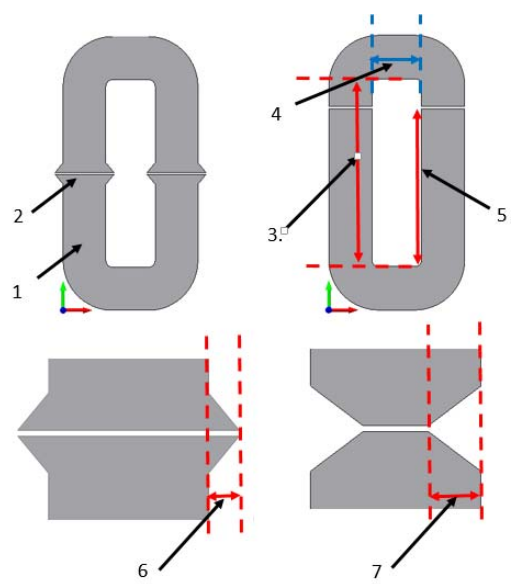

Fig. 2. Explanation of terms

Fringing flux is the main contributor to the high losses around the air gap. If the air gap area was made wider, as in Fig. 3b, this would help guide the flux away from the windings and reduce saturation at the air gap. A narrower air gap area, as in Fig. 3c, would mean that fringing fluxes had a larger air barrier between the core and winding, potentially shielding the winding from the fringing fluxes. These are in comparison to the baseline traditional core shown in Fig. 3a. Both options offer potential advantages but it was not immediately clear what side effects these modifications would have on the inductor performance and therefore which of these would be more beneficial.
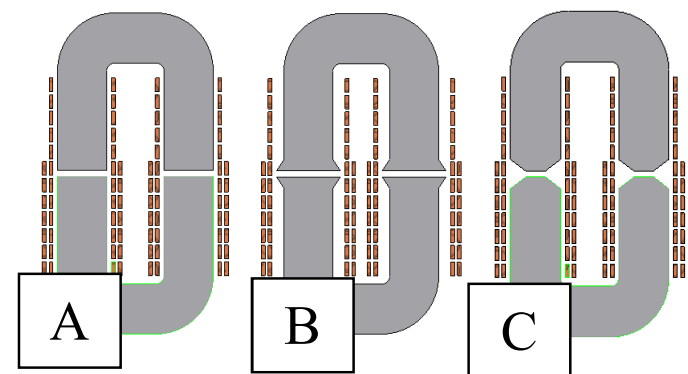

Fig. 3. Width of Core Air gap a) Standard core, b) Wider Core, positive offset and c) Narrower Core, negative offset

Moving the air gap to a different location in the core is unlikely to result in lower losses, however moving the hot spot to a more thermally accessible location would result in lower operating temperatures. Some examples of the most interesting locations are given in Fig. 4.

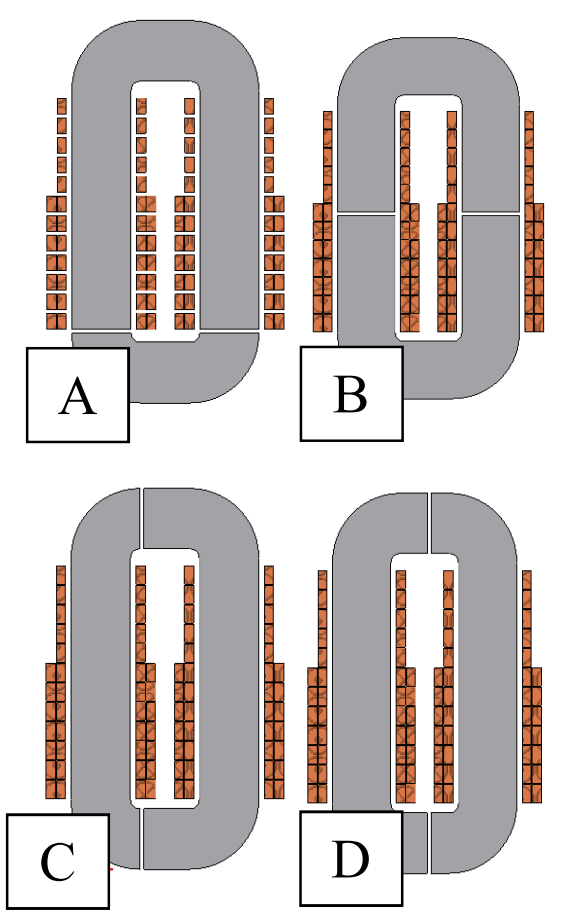

Fig. 4. Placement of air gap. a) Base of slot length b) Traditional location. c) Base of slot width d) Middle of slot width.

\section{EleCtromagnetic Results}

\section{A. Air Gap Width Offset}

Given in Fig. 5 are the flux path contour plots for the modification to the width of the air gap, although all show 
leakage flux, the fringing flux significantly varies between the widths. There is a small amount of fringing flux in the standard core, which can be seen intercepting the windings. The wider core shows no fringing flux. With the narrow core there is much more fringing flux, although most of it is contained within the air gap there is still a considerable amount intercepting the windings.

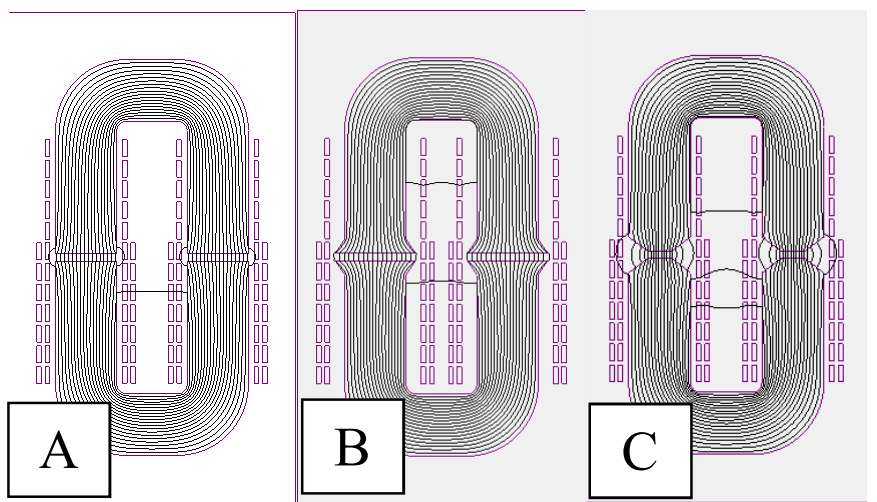

Fig. 5. Flux path for widths of Core Air gap. a) Standard core. b) Wider Core. c) Narrower Core

The effects in the change of air gap width are shown in Fig. 6, with $0 \mathrm{~mm}$ on the $\mathrm{x}$ axis representing a standard core, negative numbers are the amount cut away from both sides of the core whilst positive amounts are added, as previously shown in Fig. 2. A wider air gap is shown to have an increase in the inductance, with a narrower gap reducing inductance. This is due to the levels of total flux in the core at each width, as can be seen in Fig. 7. The flux density in the air gap stays relatively constant throughout the widths, causing the total flux to be proportional to the air gap width. With a total flux dependant on the air gap width, the flux density in the fixed width of the tooth is also proportional to the air gap width in Fig. 7, while operating below saturation. The originally designed core was limited thermally and so approaching saturation was not an issue.
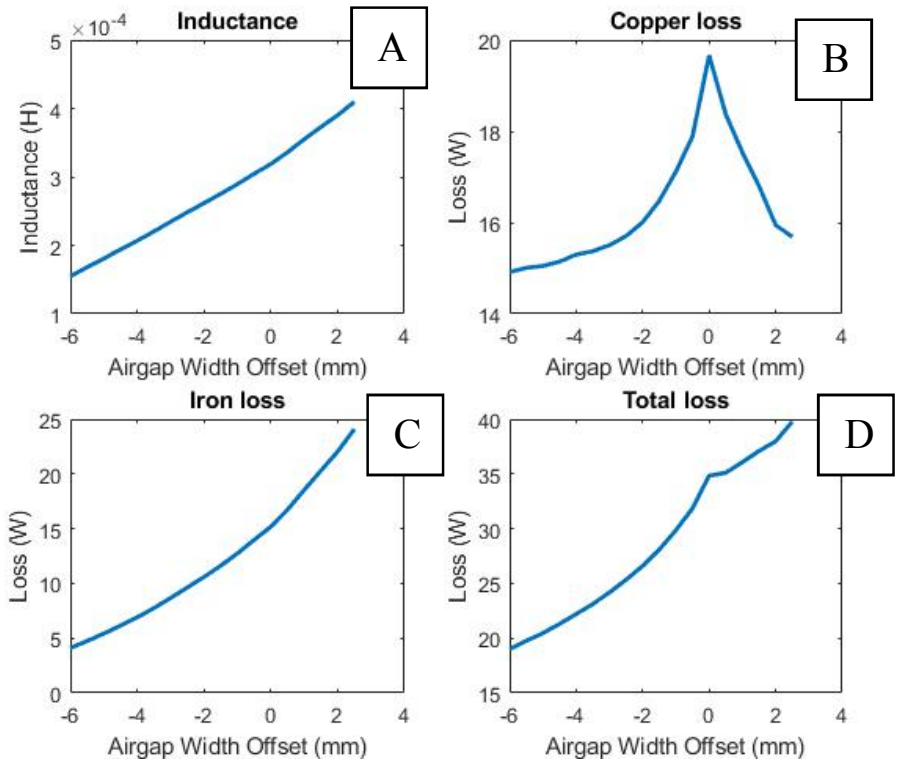

Fig. 6. Inductance and loss effects of air gap width. A) Inductance. B) Copper Loss. C) Core Loss. D) Total Loss

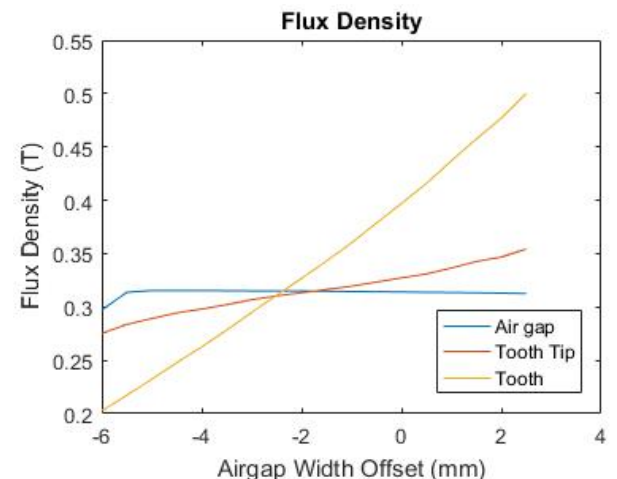

Fig. 7. Flux density change with air gap width

These changes in flux density levels also have a direct effect on the iron loss of the inductor core, shown in Fig. 6b. The formula for the loss is given by the Steinmetz equation, in equation (1). Where $P_{h}$ is the hysteresis loss per kilogram, $f$ is the frequency and $B$ is the flux density, with the constants found from the suppliers data sheet [9].

$$
P_{h}=0.00019 f^{1.51} B^{1.74}
$$

The copper losses, Fig. 6c, are at a maximum with no offset, then decrease with a wider offset, due to lower fringing flux, or a narrower offset due to reduced fringing flux intercepting the windings compared to the conventional width. The formula for the loss given per unit in the conductors due to the fringing flux is given in equation (2), with the components of $\mathrm{H}_{\perp}$ defined by equation (3) and equation (4) [10]. Where $P_{c u \text { fringe }}$ is the power loss per unit length of conductor, $\rho$ is the conductor resistivity, $\mu_{0}$ is the permeability of free space, $f$ is the frequency, $w$ the conductor width and $t$ the conductor thickness. For equation (3) and equation (4), $H_{g}$ is the air gap magnetic field, $x$ is the distance between air gap and conductor in the $\mathrm{x}$-axis direction, while $y$ is the distance in the $y$ axis direction, $l_{g}$ is the length of the air gap.

$$
\begin{gathered}
P_{c u_{-} \text {fringe }}=\frac{1}{6 \rho}\left(\pi \mu_{0} H_{\perp} f\right)^{2} w^{3} t \\
H_{x}(x, y)=\frac{H_{g}}{2 \pi} \ln \left[\frac{x^{2}+\left(y-l_{g}\right)^{2}}{x^{2}+\left(y+l_{g}\right)^{2}}\right] \\
H_{y}(x, y)=-\frac{H_{g}}{\pi}\left\{\tan ^{-1}\left[\frac{2 x l_{g}}{x^{2}+y^{2}-l_{g}^{2}}\right]+m \pi\right\}
\end{gathered}
$$

Changing the width of the air gap effects $H_{g}, l_{g}, x$ and $y$. For example when considering the extreme points of this, a width offset of $-6 \mathrm{~mm}$ gives $26 \%$ of the fringing flux losses in comparison to a traditional air gap, while the $+2 \mathrm{~mm}$ offset gives $73 \%$ of the fringing flux losses of the traditional air gap. The remaining copper losses are due to the typical winding losses (DC component, skin effect and proximity effect).

However these results can be misleading, as it is not effective to reduce losses if inductance is also reduced. So Fig. 8 takes this into account. The inductance shown here is the per unit 
inductance, with the base of $0 \mathrm{~mm}$ width offset being an inductance value of 1 . The losses are per unit but also per inductance value at that given width offset. The equations for these two calculations are given in equation (5) used for the inductance and equation (6) used for each of the losses. Where $L_{p u}$ is the inductance per unit, $L_{o f f s e t}$ is the inductance value at the current width offset and $L_{0}$ is the inductance value with no offset. Similarly $P_{\text {pup }}$ is the loss per unit per inductance, $P_{\text {offset }}$ is the loss value at the current width offset and $P_{0}$ is the loss value with no offset.

$$
\begin{gathered}
L_{p u}=\frac{L_{\text {offset }}}{L_{0}} \\
P_{\text {pup } L}=\frac{P_{o f f s e t}}{P_{0}} \times \frac{L_{o f f s e t}}{L_{0}}
\end{gathered}
$$

Fig. 8c shows that even with normalisation, the core iron losses still increase with wider offsets, as inductance increases linearly yet loss increases as a polynomial with increasing flux density. However an interesting effect can be seen with the copper losses, Fig. 8b, while they still reduce with a wider core, there is initially a slight reduction with a narrower core, then a significant increase. This results in wider cores giving lower total losses than a traditional core and a slightly narrower core also giving lower losses for a fixed inductance.

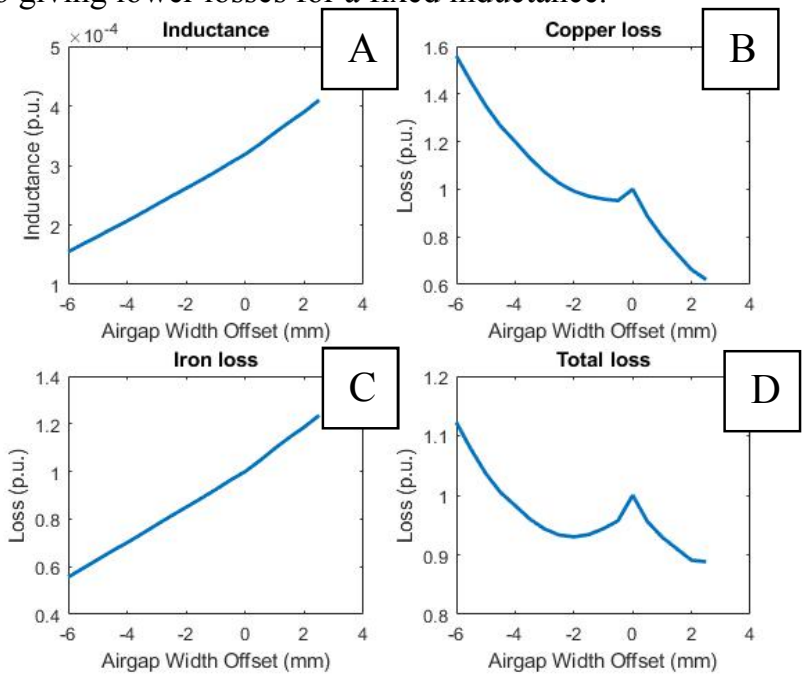

Fig. 8. Per unit inductance and loss effects of air gap width A) Inductance. B) Copper Loss. C) Core Loss. D) Total Loss

\section{B. Air Gap Placement Offset}

Considering the movement of the air gap placement, Fig. 9 shows the flux paths for the same 4 positions displayed in Fig. 4. Fig. 9 shows the position of the air gap does not have an effect on the quantity of the fringing flux. It only affects where the fringing occurs and which windings are intercepted by flux. Fig. 10 shows the effect of the air gap placement on the inductance and losses. The dashed vertical lines represent key points, with 0 being position A in Fig. 4 and Fig. 9, the red line is position $\mathrm{B}$ (traditional position), yellow is position $\mathrm{C}$ (when it first moves to the slot width, i.e. the top and bottom of the core) and purple is $\mathrm{D}$ (middle of the slot width, i.e. the top and bottom of core).

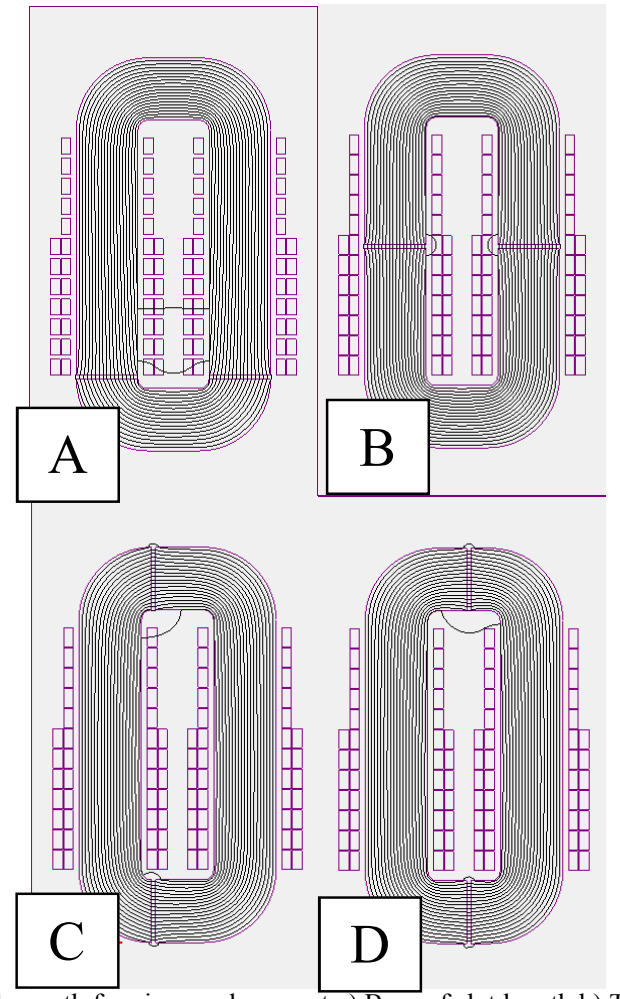

Fig. 9. Flux path for air gap placement a) Base of slot length b) Traditional location. c) Base of slot width d) Middle of slot width.
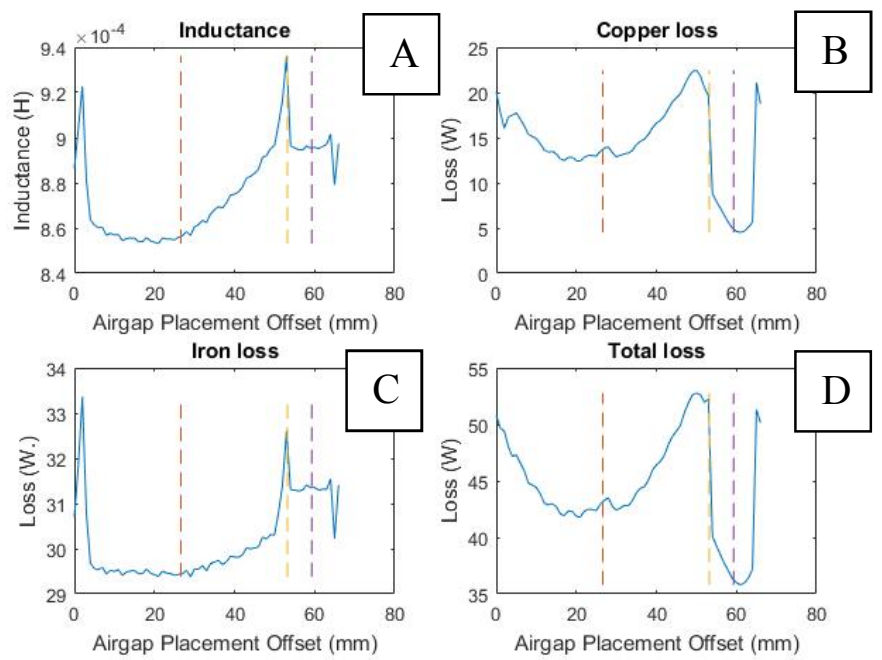

Fig. 10. Inductance and loss effects of air gap placement

While there is a change in inductance, with spikes at the extreme positions, this variation is minimal, $<10 \%$. The spikes are when the air gap is on the corner arc of the core, changing the width of the air gap, which has already been shown to have an effect on the inductance. The iron core losses mirror the inductance, again negligible change with spikes when the air gap passes the corner arcs.

The copper losses show significant deviations, with the traditional location being the lowest loss on the slot length leg (left and right of Fig. 4 and Fig. 9), however this value drops $38 \%$ when the air gap is in the middle of the slot width (top and bottom of Fig. 4 and Fig. 9). The U shape of copper losses seen 
along the slot length positioning is due to, at the extreme ends, the fringing fluxes being condensed through a smaller group of windings, this is even worse at the upper of the slot length where only a single layer of windings are present. The copper losses were much lower with the air gap along the slot width, as the fringing flux losses are dependent on the distance from air gap to winding. The same formula for the loss given per unit in the conductors due to the fringing flux, (2), can be used. With this it can be seen that, with the air gap in the middle of the slot width, position $\mathrm{D}$, the nearest conductor is $x=2.2 \mathrm{~mm}$ and $y=3.7 \mathrm{~mm}$, compared to the values at the traditional, $x=1.2 \mathrm{~mm}$, $y=0 \mathrm{~mm}$. This difference in $x$ and $y$ reduces the fringing losses to $10 \%$ of their original value, essentially leaving just the typical copper losses (DC component, skin effect and proximity effect). This type of benefit could be achieved from such methods as winding the conductors so that they are not adjacent to the air gap or utilising a thicker insulation layer between core and winding, as explained in [11], but is described here to explain the difference in copper losses observed.

\section{THERMAL MODELLING}

As the goal is to reduce the temperature of the core, the thermal model must be considered. The housing design of the components had the inductors impregnated in a potting compound (Robnor Resins PX439XS) within a small aluminium box sized for the component. The base of this aluminium box was then water cooled. As this is for automotive applications the cooling fluid is at $105^{\circ} \mathrm{C}$.

For this a lumped parameter model was used. While more advanced methods are available, such as thermal FEA, this is sufficiently accurate for this application [12-14]. For high accuracy a high node number model was used, with multiple slices throughout the core, for accurate distribution of the losses.

\section{A. Air Gap Width Offset}

Fig. 11 shows the effect of changing the air gap width on the temperature. This appears promising for a reduction in air gap width, but from before, this also had a much lower inductance. Fig. 12 shows the temperature for a fixed inductance. This shows, as was seen with the losses, when the inductance achieved is taken into account, the wider air gap has superior performance.

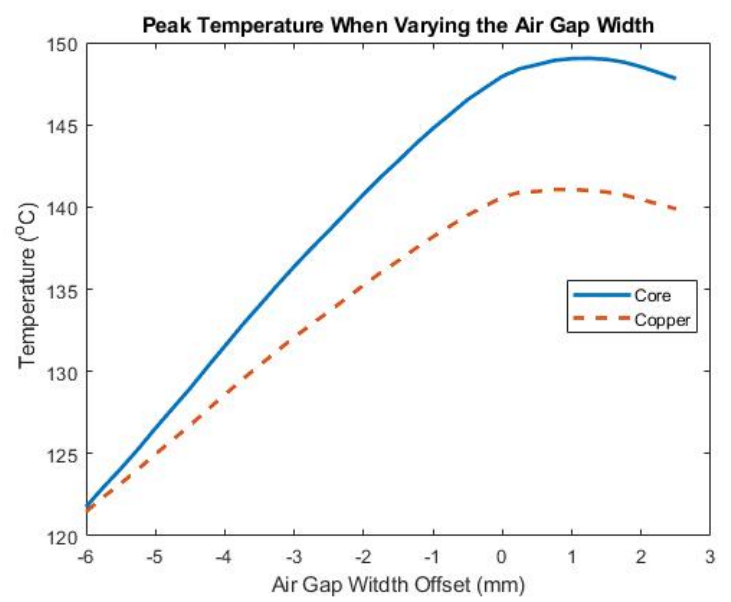

Fig. 11. Thermal results with air gap width offset range

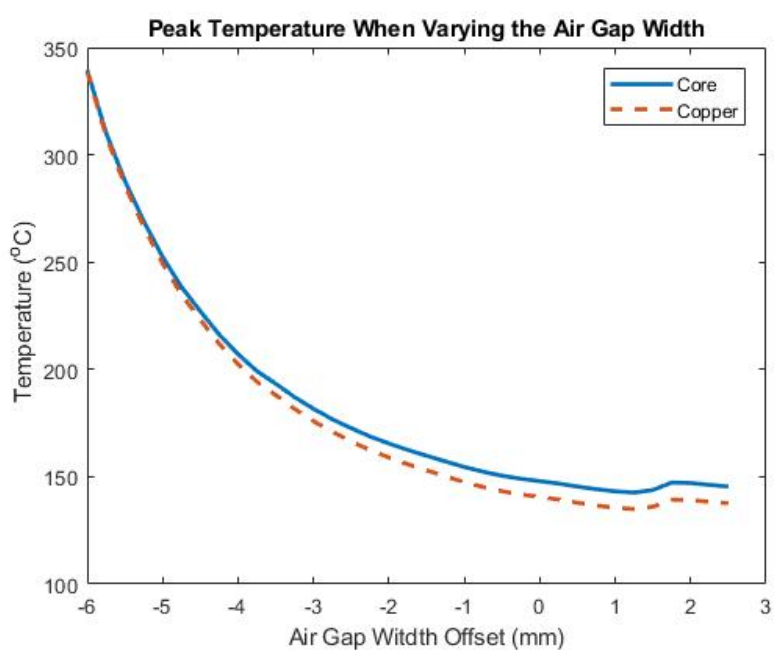

Fig. 12. Thermal results with air gap width offset range for a fixed inductance

\section{B. Air Gap Placement Offset}

Fig. 13 gives the change in temperature with the movement of the air gap. It can be seen that there is correlation with the losses, as expected, but also significantly increases as the air gap moves to less thermally accessible areas. Here it can be clearly seen that moving the air gap to the slot width, the top and bottom of the $U$ cores, has significant benefits to the thermal performance of the inductor.

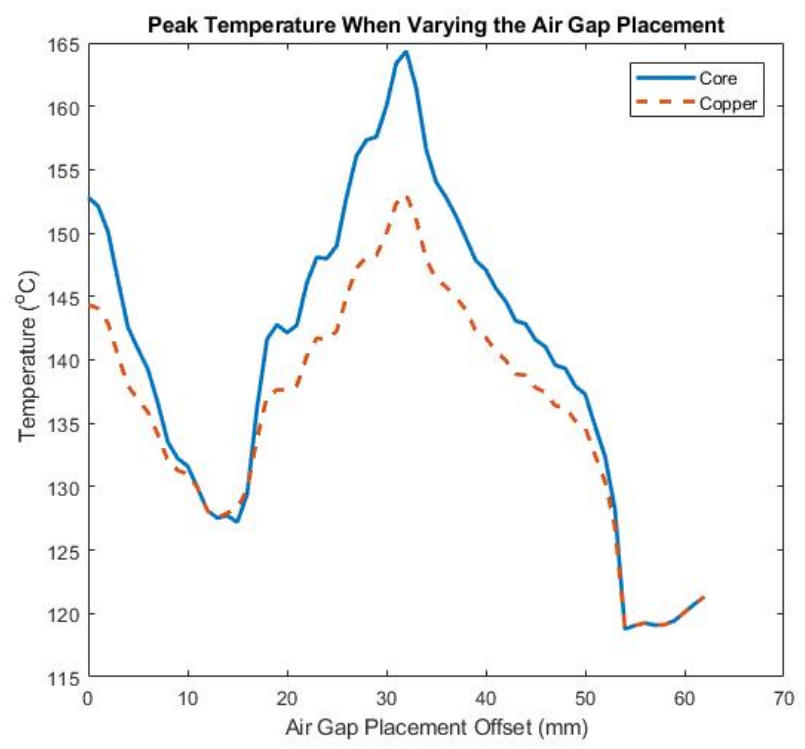

Fig. 13. Thermal results with air gap placement offset range

\section{CONCLUSIONS AND FURTHER WORK}

The advancement of additive manufacturing for magnetic cores is allowing for more freedom in the design of inductors. One critical area for this is investigating the effects of changing the size or placement of the air gap that would have been difficult to manufacture previously. Increasing the width of the air gap can lead to lower copper and total losses for a fixed inductance, but reduces the potential slot area. While this is not an issue with this case study, as adequate fill factor is still achievable, in 
more packed applications it could be a limitation. Reducing the air gap width can lead to lower core losses and copper losses, provided the reduction in width is not too extreme.

Moving the air gap has little effect on the inductance but can significantly reduce the copper loss. The main benefit of moving the air gap is moving the potential hot spot to a more thermally accessible locations.

The future work is to design inductors for the original specification, with the thermal benefits shown. This will show how beneficial these changes to the core are in terms of mass and volume savings. Longer term, additively manufactured inductors will be tested with these core modifications applied. Before that, laminated cores manufactured through electrical discharge machining will be characterised to reinforce the performance of the proposed designs. This will highlight any winding difficulties caused by the new core shapes, so they can be overcome. At the same time, simple core shapes are being $3 \mathrm{D}$ printed to establish potential performance differences between printed cores and off the shelf cores.

\section{REFERENCES}

[1] S. Kimura, Y. Itoh, W. Martinez, M. Yamamoto, and J. Imaoka, "Downsizing Effects of Integrated Magnetic Components in High Power Density DC\&\#x2013;DC Converters for EV and HEV Applications," IEEE Transactions on Industry Applications, vol. 52, pp. 3294-3305, 2016.

[2] J. Scoltock, G. Calderon-Lopez, Y. Wang, and A. J. Forsyth, "Design optimisation and trade-offs in multikW DC-DC converters," in 2016 IEEE Energy Conversion Congress and Exposition (ECCE), 2016, pp. 1-8.

[3] C. W. T. McLyman, Transformer and inductor design handbook, 4th ed. Boca Raton, FL: CRC Press, 2011.

[4] (2016). Magnetics - Kool Mu Cores. Available: https://www.mag-inc.com/Products/Powder-

Cores/Kool-Mu-Cores.aspx

[5] L. Liu, C. Ding, S. Lu, T. Ge, Y. Yan, Y. Mei, et al., "Design and additive manufacturing of multi- permeability magnetic cores," in 2017 IEEE Energy Conversion Congress and Exposition (ECCE), 2017, pp. 881-886.

[6] Y. Yan, D. Chao, K. D. T. Ngo, Y. Mei, and G. Q. Lu, "Additive manufacturing of planar inductor for Power Electronics applications," in 2016 International Symposium on 3D Power Electronics Integration and Manufacturing (3D-PEIM), 2016, pp. 1-16.

[7] Y. Yan, J. Moss, K. D. T. Ngo, Y. Mei, and G. Q. Lu, "Additive manufacturing of toroid inductor for power electronics applications," in 2016 IEEE Energy Conversion Congress and Exposition (ECCE), 2016, pp. 1-6.

[8] R. Wrobel and P. H. Mellor, "Thermal Design of HighEnergy-Density Wound Components," IEEE Transactions on Industrial Electronics, vol. 58, pp. 4096-4104, 2011.

[9] HitachiMetals. (2012). Powerlite Inductor Cores Technical Bulletin.

[10] W. A. Roshen, "Fringing Field Formulas and Winding Loss Due to an Air Gap," IEEE Transactions on Magnetics, vol. 43, pp. 3387-3394, 2007.

[11] M. K. Kazimierczuk, High-frequency magnetic components, Second edition. ed. Chichester, Sussex: John Wiley \& Sons, 2014.

[12] A. Boglietti, A. Cavagnino, D. Staton, M. Shanel, M. Mueller, and C. Mejuto, "Evolution and Modern Approaches for Thermal Analysis of Electrical Machines," IEEE Transactions on Industrial Electronics, vol. 56, pp. 871-882, 2009.

[13] C. Sciascera, P. Giangrande, L. Papini, C. Gerada, and M. Galea, "Analytical Thermal Model for Fast Stator Winding Temperature Prediction," IEEE Transactions on Industrial Electronics, vol. 64, pp. 6116-6126, 2017.

[14] R. Wrobel, N. McNeill, and P. H. Mellor, "Performance Analysis and Thermal Modeling of a High-Energy-Density Prebiased Inductor," IEEE Transactions on Industrial Electronics, vol. 57, pp. 201-208, 2010. 